\title{
Tratamento cirúrgico do pé eqüino na paralisia cerebral: uma revisão sistemática e quantitativa da literatura*
}

\author{
Surgical treatment of equinus varus deformity \\ of the foot in cerebral palsy: a systematic and \\ quantitative review of the literature
}

Rodrigo Montezuma César de Assumpção ${ }^{1}$, Patricia Maria de Moraes Barros Fucs², Celso Svartman ${ }^{3}$

\section{RESUMO}

Objetivo: Realizar uma revisão sistemática e quantitativa da literatura que versa sobre o tratamento cirúrgico do pé equiino na paralisia cerebral, submetendo os resultados à apreciação estatística, caracterizando-se assim uma metanálise, cujo propósito é esclarecer qual a técnica cirúrgica que apresenta o menor risco de recidiva da deformidade em equiino e quando devemos operar. Métodos: Revisão sistemática e quantitativa da literatura

* Trabalho realizado no Grupo das Doenças Neuromusculares do Departamento de Ortopedia e Traumatologia da Santa Casa de Misericórdia de São Paulo - Pavilhão "Fernandinho Simonsen", Faculdade de Ciências Médicas da Santa Casa de São Paulo São Paulo (SP), Brasil.

1. Mestre; Chefe do Grupo das Doenças Neuromusculares; Faculdade de Ciências Médicas da Santa Casa de São Paulo - São Paulo (SP), Brasil.

2. Doutor, Professor Adjunto da Faculdade de Ciências Médicas da Santa Casa de São Paulo; Consultora do Grupo das Doenças Neuromusculares - São Paulo (SP), Brasil.

3. Doutor, Professor Assistente, Faculdade de Ciências Médicas da Santa Casa de São Paulo, Consultor do Grupo das Doenças Neuromusculares - São Paulo (SP), Brasil.

Endereço para correspondência: Santa Casa de Misericórdia de São Paulo, Departamento de Ortopedia e Traumatologia, Rua Dr. Cesário Motta Jr., 112 - 01227-900 - São Paulo (SP), Brasil. Tel./fax: (11) 2176-1566. E-mail: dot.neuromuscular@santacasasp.org.br Recebido em 27/5/08. Aprovado para publicação em 30/9/08. Copyright RBO2008 entre 1966 e 2007. A seleção dos artigos seguiu critérios de inclusão e exclusão preestabelecidos. Os estudos foram identificados e escolhidos utilizando como estratégia de busca os bancos de dados computadorizados da Internet (Medline, Embase, Lilacs e Biblioteca Virtual Cochrane). Também foi realizada a busca manual de estudos em periódicos e livros. Os dados de cada estudo foram coletados conforme formulário preestabelecido. Foi avaliada a qualidade metodológica de cada estudo. Calculou-se para cada estudo selecionado a estimativa do efeito do tratamento (risco relativo de recidivas). Os dados foram submetidos à apreciação estatística específica direcionada para revisões sistemáticas. Os estudos e dados obtidos foram submetidos à análise clínica e estatística de heterogeneidade por meio da análise de subgrupos (média de idade, tempo de seguimento e técnica cirúrgica utilizada). $R e$ sultados: Do total de 70 publicações encontradas, foram selecionados 20 estudos observacionais retrospectivos. A análise dos estudos com seguimento médio acima de cinco anos mostra que as taxas de recidiva se tornam de maior valor absoluto quanto maior o tempo de seguimento. Os estudos em que a média de idade dos pacientes no momento da cirurgia foi superior aos sete anos mostram menor soma dos riscos relativos, quando comparados ao grupo com média de idade inferior aos sete anos, 
evidenciando diminuição significativa do risco absoluto. A análise comparativa dos estudos que citam alongamentos realizados no tendão calcâneo mostra soma dos riscos relativos inferiores, quando comparados aos estudos que citam alongamentos na junção músculo-tendão, porém, com diminuição pequena no risco absoluto. Conclusões: A zetaplastia para o alongamento do tendão calcâneo tende a ser mais segura. Os pacientes devem ser operados após os sete anos de idade. A longo prazo, o risco de recidiva aumenta de forma significativa, reforçando a necessidade do seguimento destes pacientes, no mínimo, até a maturidade esquelética.

Descritores - Paralisia cerebral/complicações; Pé eqüino/ etiologia; Pé eqüino/cirurgia; Procedimentos ortopédicos/métodos; Metanálise

\section{ABSTRACT}

Objective: To perform an systematic and quantitative review of the literature dealing with the surgical treatment of equinus foot in cerebral palsy, to perform a statistical analysis of the results in order to characterize a meta-analysis, the purpose of which is to clarify which of the surgical techniques has the lesser risk of recurrence of the equinus deformity, and when to operate on. Methods: Systematic and quantitative review of the literature between 1966 and 2007. The selection of articles followed a preestablished inclusion and exclusion criteria. The studies were identified and chosen using the computer databases in the Internet as the search strategy (Medline, Embase, Lilacs, and Cochrane Virtual Library). A manual search in journals and books was also done. The data of each study were collected in a pre-established form. The methodological quality of each study was evaluated. For each study selected an estimate was made of the effects of the treatment (relative risk of recurrence). The data were submitted to clinical analysis and to statistical heterogeneity appreciation by analyzing subgroups (mean age, duration of follow-up, and surgical technique used). Results: From the 70 publications found, 20 observational, retrospective studies were selected. Analysis of the studies with mean follow-up longer than five years shows that the rate of recurrence are of higher absolute value in proportion with the longer duration of the follow-up. The studies in which the mean age of patients at the time of surgery was older than seven years show a smaller sum total of relative risks when compared to the group with mean ager younger than seven years, showing a significant reduction of the absolute risk. The comparative analysis of studies that mention performance of lengthening of the calcaneus tendon shows a lower sum total of risks when compared to studies that mention lengthening in the muscle-tendon junction, but with a small reduction in absolute risk. Conclusions: Zetaplasty for the lengthening of the calcaneus tendon tends to be safer. Patients should operated on after seven years of age. In the long term, the risk of recurrence increases significantly, which reinforces the need for a follow-up of these patients at least till they reach skeletal maturity.

Keywords - Cerebral palsy/complications; Equinus deformity/etiology; Equinus deformity/ surgery; Orthopedic procedures/methods; Meta-analysis

\section{INTRODUÇÃO}

O pé eqüino é a deformidade mais comum que requer tratamento no paciente com paralisia cerebral. Foi estimado que $20 \%$ a $25 \%$ de todos os pacientes vão precisar algum procedimento cirúrgico para este tipo de deformidade ${ }^{(1)}$.

O tratamento cirúrgico está indicado nos pacientes em que o tratamento não cirúrgico falhou e nas deformidades fixas, não redutíveis à dorsiflexão passiva do tornozelo. Deve-se levar sempre em conta a idade na qual o procedimento está sendo indicado, pois as recidivas são mais freqüentes nos pacientes abaixo dos quatro anos de idade ${ }^{(2-3)}$. Várias técnicas cirúrgicas foram propostas: denervação dos músculos gastrocnêmios e/ou sóleo, alongamento isolado dos gastrocnêmios, ressecção das origens dos gastrocnêmios, alongamentos do tendão calcâneo e a sua translocação para posição anterior no calcâneo ${ }^{(4)}$. Todos foram criados para reduzir o reflexo do estiramento exacerbado e alongar o músculo. Todos enfraquecem o músculo. $\mathrm{O}$ 
alongamento do tendão calcâneo aumenta a excursão do conjunto gastrocnêmio-sóleo, logo o objetivo principal é evitar alongá-lo em demasia e diminuir a taxa de recidiva ${ }^{(4)}$.

O procedimento ideal deve ser seguro e eficaz, restaurando e mantendo o alongamento apropriado do músculo. Não deve causar encurtamentos (recidiva da deformidade) com o crescimento ou o alongamento em excesso (deformidade em calcâneo) com o aumento do peso corpóreo e da estatura ${ }^{(5)}$.

O melhor tratamento ou a combinação ideal de técnicas ainda não existe. Os níveis de evidência e graus de recomendação dos estudos existentes atualmente na literatura, que se referem ao tratamento cirúrgico do pé equiino na paralisia cerebral espástica, são trabalhos do tipo análise de resultados e séries de casos, estudos não controlados, não randomizados e revisões narrativas $^{(5)}$.

Realizou-se revisão sistemática da literatura pertinente submetendo os resultados a apreciação estatística, caracterizando-se assim uma metanálise. Nosso principal objetivo foi esclarecer o seguinte ponto: Qual técnica cirúrgica apresenta o menor risco de recidiva da deformidade em eqüino dos pacientes portadores de paralisia cerebral espástica? Qual técnica cirúrgica apresenta o maior risco de deformidade em calcâneo? Qual é a idade ideal para realizar os procedimentos cirúrgicos? Até quando devemos seguir ambulatorialmente os pacientes?

\section{MÉTODOS}

Realizou-se revisão sistemática e quantitativa da literatura publicada entre 1966 e 2007, que versa sobre o tratamento cirúrgico do pé eqüino na paralisia cerebral. O trabalho foi realizado no Departamento de Ortopedia e Traumatologia da Santa Casa de Misericórdia de São Paulo, com aprovação do Comitê de Ética desta instituição.

Este estudo tem como prioridade responder à seguinte questão: Qual é a técnica cirúrgica mais eficaz no tratamento do pé eqüino dos pacientes portadores de paralisia cerebral espástica? A eficácia será medida quanto às taxas de recidiva e de deformidade em calcâneo.
Realizamos a seleção dos artigos segundo os seguintes critérios de inclusão e exclusão: 1. Foram incluídos estudos primários analíticos observacionais (coorte retrospectivos e prospectivos) e experimentais (estudos randomizados controlados e não controlados). Foram excluídos relatos de caso, editoriais, descrições de técnicas cirúrgicas e revisões narrativas; 2. Foram incluídos estudos realizados a partir de 1966; 3. Foram incluídos estudos publicados em todos os idiomas; 4. Foram excluídas publicações múltiplas de uma mesma população em estudo; 5. Estipulamos restrições para os tamanhos das amostras e seu seguimento: 20 pacientes com seguimento mínimo de dois anos; 6 . Foram incluídos estudos que reportam o tratamento cirúrgico da deformidade em equiino do tornozelo de pacientes portadores de paralisia cerebral espástica. Os tratamentos devem ter efeitos similares sobre os resultados dos estudos analisados; 7. Os resultados foram medidos por meio da análise das taxas de recidiva e deformidade em calcâneo; e 8. Só incluímos como elegíveis estudos publicados e completos.

Os estudos foram identificados e selecionados por um revisor (autor principal), utilizando como estratégia de busca e fonte de informação os seguintes bancos de dados computadorizados na Internet de 1966 a 2007: Medline, Lilacs, Embase e Biblioteca Virtual Cochrane. As palavras-chave para busca foram: "tratamento cirúrgico", "pé eqüino", "paralisia cerebral”. Também foi realizada a busca manual de estudos em periódicos e livros.

Os dados foram coletados pelo autor, de cada estudo, conforme formulário próprio.

O processo de coleta de dados, inclusão e exclusão dos estudos foi realizado duas vezes pelo autor principal.

A análise dos dados foi realizada baseando-se em um modelo de efeitos fixos, em que a inferência estatística é condicionada a estudos já realizados, isto é, retrospectivos. Para tal aplicaram-se os métodos estatísticos de Mantel-Haenzel(6) e o método baseado na variância geral utilizando intervalos de confiança. Calculou-se para cada estudo selecionado a estimativa do efeito, isto é, o efeito do tratamento da seguinte forma: para estudos em que havia grupos-controle esco- 
lheu-se a razão de probabilidades (odds ratio) e para aqueles sem grupo-controle, o cálculo do risco relativo.

Assumiu-se que os grupos-controle desses estudos, para fins de cálculo, seriam representados por pacientes portadores da deformidade em eqüino, porém não tratados e assim sendo igual ao tamanho total da amostra de pacientes ${ }^{(7)}$. Foi também calculado o intervalo de confiança para as proporções encontradas. Para os dois subgrupos calculou-se a soma estatística dos efeitos e dos intervalos de confiança. Os cálculos e seus resultados foram conferidos e refeitos, utilizando-se o programa estatístico Stata $8.0^{(8)}$.

Estudos observacionais e não experimentais sobre um mesmo tópico são sempre divergentes na sua arquitetura, nos métodos para coleta de dados, nos seus desfechos e no grau de controle de vieses. Calcular uma simples soma da estimativa do efeito, frente a tamanha diversidade pode gerar falsa visão do problema; assim, decidiu-se realizar também a análise da heterogeneidade destes estudos não experimentais. Dessa forma, procedemos à análise da heterogeneidade clínica e metodológica. Clinicamente, detectou-se a di- visão dos estudos naqueles com e sem grupo-controle, média de idade, seguimento médio e técnica cirúrgica. $\mathrm{Na}$ análise metodológica utilizou-se o método estatístico baseado na variância geral, calculando-se o valor de Q (distribuição qui-quadrada com os graus de liberdade - número de estudos menos um) para os grupos de estudos escolhidos. Fixou-se o valor de significância em 0,05 para a rejeição da hipótese de homogeneidade dos estudos.

\section{RESULTADOS}

Encontramos um total de 70 publicações e dentre estas foram selecionados 20 estudos observacionais do tipo coorte retrospectivos, totalizando 1.250 pacientes portadores de paralisia cerebral espástica e deformidade em equiino do pé. Não se encontrou estudo experimental randomizado e controlados sobre o assunto em questão.

Cinqüenta estudos foram excluídos.

As características básicas de cada estudo incluído na revisão estão descritas na tabela 1 .

TABELA 1

Variáveis básicas coletadas dos estudos incluídos na revisão sistemática

\begin{tabular}{|c|c|c|c|c|c|c|c|}
\hline Autor principal & $\begin{array}{c}\text { Ano de } \\
\text { publicação }\end{array}$ & $\begin{array}{c}\text { Seguimento } \\
\text { médio } \\
\text { (meses) }\end{array}$ & $\begin{array}{c}\text { Tamanho } \\
\text { da amostra }\end{array}$ & $\begin{array}{c}\text { Tamanho } \\
\text { da amostra } \\
\text { grupo } \\
\text { controle }\end{array}$ & $\begin{array}{l}\text { Idade } \\
\text { (meses) }\end{array}$ & $\begin{array}{l}\text { Técnica } \\
\text { cirúrgica } \\
\text { utilizada }\end{array}$ & $\begin{array}{c}\text { Técnica } \\
\text { cirúrgica } \\
\text { grupo } \\
\text { controle }\end{array}$ \\
\hline Lemperg $^{(13)}$ & 1969 & 48 & 48 & 43 & 72 & Z & Sv \\
\hline Sharrard ${ }^{(24)}$ & 1972 & 105 & 77 & 53 & 82 & Z & V e St \\
\hline Pierrot $(25)$ & 1974 & 42 & 32 & 22 & 78 & $\mathrm{HCA}$ & Wh e Z \\
\hline Craig ${ }^{(17)}$ & 1976 & 72 & 100 & - & 96 & St e Wh & \\
\hline Cigala & 1979 & 42 & 20 & - & 84 & $\mathrm{Bk}$ & \\
\hline $\operatorname{Lee}^{(3)}$ & 1980 & 24 & 71 & 51 & 72 & Hoke & Bk e St \\
\hline Gaines $^{(27)}$ & 1984 & 42 & 68 & - & 77 & Z & \\
\hline Garbarino(10) & 1985 & 36 & 26 & - & 106 & Z & \\
\hline Grant $^{(15)}$ & 1985 & 120 & 39 & - & 54 & TAL & \\
\hline $\operatorname{Javros}^{(28)}$ & 1987 & 67 & 79 & - & 80 & $\mathrm{~V}$ & \\
\hline Olney $^{(9)}$ & 1988 & 91 & 219 & - & 54 & $\mathrm{Bk}$ & \\
\hline Graham ${ }^{(29)}$ & 1988 & 160 & 35 & - & 81 & Wh & \\
\hline Rosenthal(30) & 1992 & 98 & 87 & - & 70 & V & \\
\hline Cheng ${ }^{(31)}$ & 1993 & 42 & 71 & - & 72 & Hoke $p$ & \\
\hline Rattey ${ }^{(16)}$ & 1993 & 115,2 & 77 & - & 64,8 & Z & \\
\hline Svartman ${ }^{(32)}$ & 1994 & 51 & 56 & - & 66 & $\overline{T A L}$ & \\
\hline Douté(18) & 1997 & 108 & 38 & - & 96 & Neurec & \\
\hline Sala ${ }^{(19)}$ & 1997 & 66 & 27 & - & 87 & Hoke & \\
\hline $\mathrm{Katz}^{(33)}$ & 2000 & 90 & 52 & - & 60 & Hoke $p$ & \\
\hline Saraph(11) & 2000 & 26,4 & 28 & - & 151 & Baum & \\
\hline Total & & 72,28 & 1.250 & & 80,2 & & \\
\hline
\end{tabular}




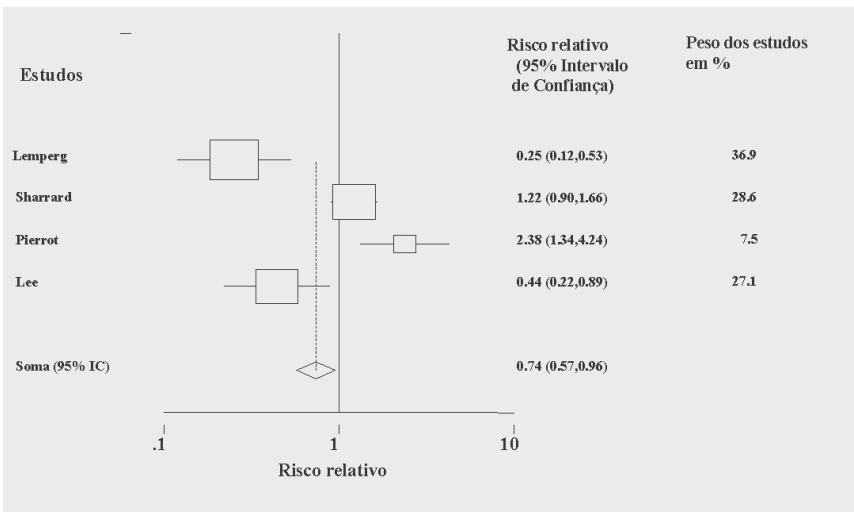

Gráfico 1 - Resultado da metanálise dos estudos com grupocontrole

Foi analisada a variável dicotômica como medida do resultado final: recidiva da deformidade em eqüino. A presença de deformidade em calcâneo não foi analisada devido à escassez de dados dos trabalhos com relação a esta complicação.

Os gráficos 1 e 2 representam o desenho esquemático da análise quantitativa dos estudos selecionados através de cálculo estatístico (metanálise). O grupo de estudos selecionados foi subdividido em dois subgrupos com e sem grupo-controle, respectivamente, contemplando assim a heterogeneidade dos estudos e tornando os subgrupos mais homogêneos.

\section{Resultados da análise da heterogeneidade}

Estudos não experimentais sobre o mesmo tópico são quase sempre adversos nos seus delineamentos, ou arquitetura, nos métodos para coletar os dados, nas definições dos desfechos e no grau de controle de vieses. Calcular um único valor médio das estimativas de efeito (risco relativo) pode levar a grosseiro erro de interpretação. A exploração da heterogeneidade representa a oportunidade de aumentar a relevância das conclusões e salientar o entendimento científico dos estudos analisados.

A heterogeneidade dos estudos foi avaliada clinicamente dividindo-os de acordo com a presença ou não de grupo-controle, idade maior e menor do que sete anos, seguimento maior e menor do que cinco anos e técnica cirúrgica (alongamento no tendão calcâneo ou na junção músculo-tendão). Estatisticamente calculan-

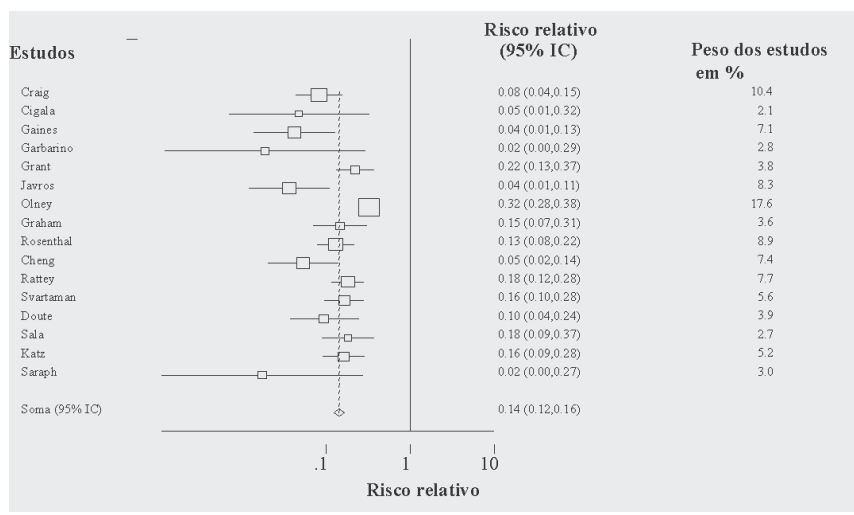

Gráfico 2 - Resultado da metanálise dos estudos sem grupocontrole

do o valor de Q (qui-quadrado) para cada estudo, o numero de graus de liberdade e o valor de $p$ fixado em 0,05 . Se este valor for inferior a 0,05 , rejeita-se a hipótese de homogeneidade. Estes valores e os cálculos estatísticos estão especificados em anexo e mostram heterogeneidade dos estudos selecionados (tabelas $2 \mathrm{e}$ $3)$.

Conforme mostram as tabelas 2 e 3 , a hipótese de homogeneidade dos estudos foi rejeitada, pois o valor de $p$ é menor do que 0,05 . Logo podemos inferir que os estudos são heterogêneos.

Continuando a análise da heterogeneidade, realizamos a análise de subgrupo por meio de gráficos, dividindo os estudos em subgrupos, a saber: seguimento (maior e menor do que cinco anos); subgrupo idade (maior e menor do que sete anos); subgrupo técnicas cirúrgicas (alongamentos no tendão calcâneo e alongamentos na junção músculo-tendão).

\section{Interpretação dos resultados}

Os resultados foram interpretados analisando-se a redução dos riscos relativos dos subgrupos seguimento médio, média de idade e técnica cirúrgica, por meio da subtração dos valores dos riscos relativos. O valor percentual da redução foi aquilatado pelo cálculo básico de percentagem. Fez-se também o cálculo do número necessário de pacientes a serem tratados em cada subgrupo, para que o evento adverso (recidiva da deformidade em equiino) fosse prevenido, dividindo-se numeral um pelo valor da diferença dos riscos relativos. 
TABELA 2

Análise dos estudos com grupo controle

\begin{tabular}{|c|c|c|c|}
\hline Estudo & $\begin{array}{l}\text { Risco } \\
\text { relativo }\end{array}$ & $\begin{array}{l}\text { [95\% intervalo } \\
\text { de conf.] }\end{array}$ & $\%$ peso \\
\hline Lemperg $^{(13)}$ & 0,25 & $0,12-0,53$ & 36,8 \\
\hline Sharrard ${ }^{(24)}$ & 1,22 & $0,90-1,66$ & 28,5 \\
\hline Pierrot ${ }^{(25)}$ & 2,38 & $1,33-4,24$ & 7,5 \\
\hline $\operatorname{Lee}^{(3)}$ & 0,44 & $0,22-0,88$ & 27,0 \\
\hline $\begin{array}{l}\text { Soma estatística dos riscos relativos } \\
\text { (método de Manthel-Haezel) }\end{array}$ & 0,74 & $0,57-0,95$ & \\
\hline \multicolumn{4}{|l|}{$\begin{array}{l}\text { Heterogeneidade, qui-quadrado }=36,21 \\
(\text { d.f. }=3) ; p=0,000\end{array}$} \\
\hline Teste do $R R=1 ; z=2,31 ; p=0,021$ & & & \\
\hline
\end{tabular}

TABELA 3

Análise dos estudos sem grupo controle

\begin{tabular}{|c|c|c|c|}
\hline Estudo & $\begin{array}{c}\text { Risco } \\
\text { relativo }\end{array}$ & $\begin{array}{l}\text { Intervalo de } \\
\text { confiança }\end{array}$ & $\begin{array}{r}\text { Peso dos } \\
\text { estudos (\%) }\end{array}$ \\
\hline Craig(17) & 0,08 & $0,04-0,15$ & 10,4 \\
\hline Cigala ${ }^{(26)}$ & 0,05 & $0,00-0,32$ & 2,1 \\
\hline Gaines $^{(27)}$ & 0,04 & $0,01-0,13$ & 7,1 \\
\hline Garbarino(10) $^{(10)}$ & 0,02 & $0,00-0,29$ & 2,8 \\
\hline Grant $^{(15)}$ & 0,22 & $0,13-0,37$ & 3,7 \\
\hline Javros $^{(28)}$ & 0,03 & $0,01-0,11$ & 8,2 \\
\hline Olney ${ }^{(9)}$ & 0,32 & $0,27-0,38$ & 17,6 \\
\hline Graham ${ }^{(29)}$ & 0,14 & $0,07-0,30$ & 3,5 \\
\hline Rosenthal(30) & 0,13 & $0,08-0,21$ & 8,9 \\
\hline Cheng $^{(31)}$ & 0,05 & $0,02-0,14$ & 7,4 \\
\hline Rattey ${ }^{(16)}$ & 0,18 & $0,12-0,28$ & 7,6 \\
\hline Svartaman ${ }^{(32)}$ & 0,16 & $0,09-0,28$ & 5,6 \\
\hline Doute $^{(18)}$ & 0,09 & $0,04-0,24$ & 3,9 \\
\hline Sala ${ }^{(19)}$ & 0,18 & $0,09-0,37$ & 2,7 \\
\hline $\mathrm{Katz}^{(33)}$ & 0,16 & $0,09-0,28$ & 5,2 \\
\hline Saraph ${ }^{(11)}$ & 0,02 & $0,00-0,27$ & 3,0 \\
\hline $\begin{array}{l}\text { Soma dos riscos relativos } \\
\text { (método de Manthel-Haezel) }\end{array}$ & 0,14 & $0,12-0,16$ & \\
\hline \multicolumn{4}{|l|}{$\begin{array}{l}\text { Heterogeneidade qui-quadrado }=132,56 \\
(\text { d.f. }=15) ; p=0,000\end{array}$} \\
\hline $\begin{array}{l}\text { Teste do risco relativo }=1 \\
z=26,89 ; p=0,000\end{array}$ & & & \\
\hline
\end{tabular}

Os estudos com seguimento médio mais longo, isto é, com mais de cinco anos, mostram que as taxas de recidiva se tornam de maior valor absoluto na razão direta do maior tempo médio de seguimento (gráfico 3). Comparando os valores das somas estatísticas dos riscos relativos, observamos aumento significativo de 


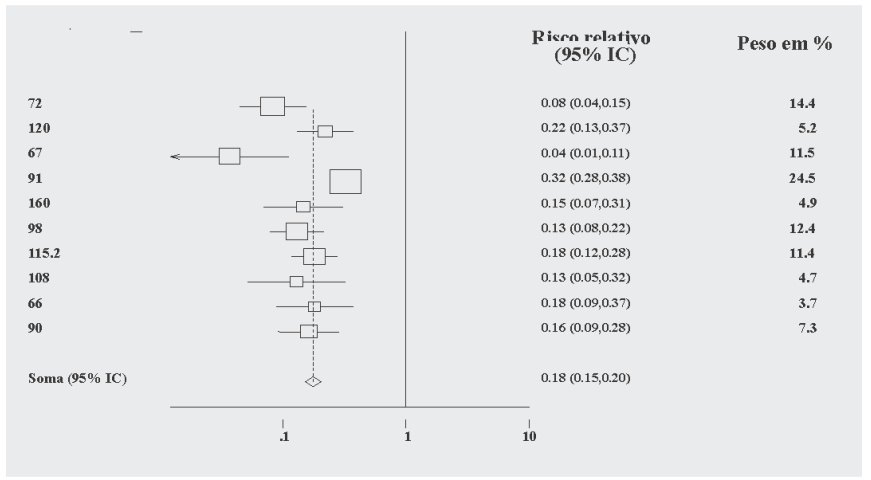

Gráfico 3 - Seguimento maior do que cinco anos

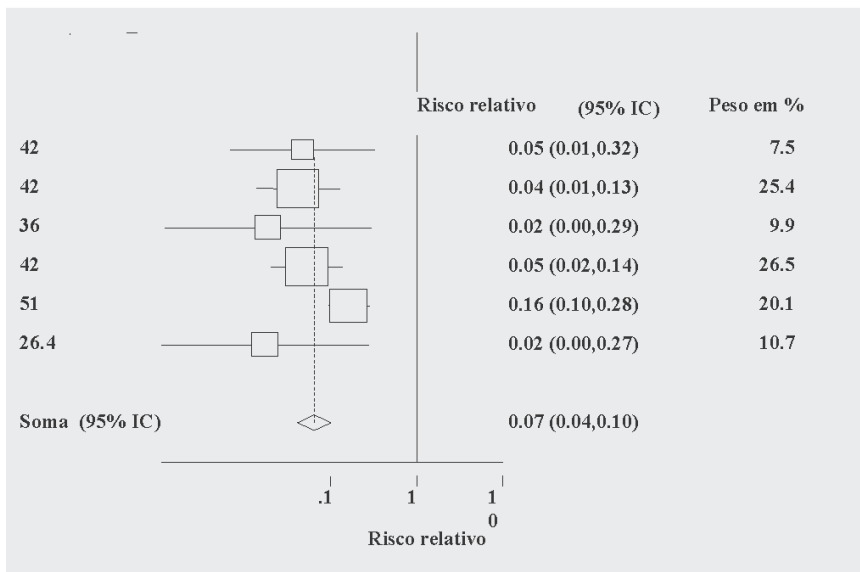

Gráfico 4 - Resultado da análise dos estudos com seguimento menor do que cinco anos

0,07 nos estudos com seguimento médio abaixo dos cinco anos (gráfico 4), e 0,18 para aqueles com seguimento médio acima dos cinco anos. A diferença percentual relativa destes valores representa uma redução relativa do risco de recidivas de $39 \%$. Em termos absolutos, este valor seria de 0,11 . Assim sendo, a cada nove pacientes operados prevenimos uma recidiva (1/ $0,11)$.

Os estudos em que a média de idade dos pacientes, no momento da cirurgia, foi superior aos sete anos mostram menor soma estatística dos riscos relativos, quando comparado com o grupo com idade média inferior aos sete anos, 0,08 e 0,16, respectivamente (gráficos 5 e 6). Demonstra-se dessa forma diminuição significativa do risco absoluto de 0,08 , uma redução percentual de $50 \%$. A cada 12 pacientes operados, prevenimos 1 recidiva $(1 / 0,08)$.

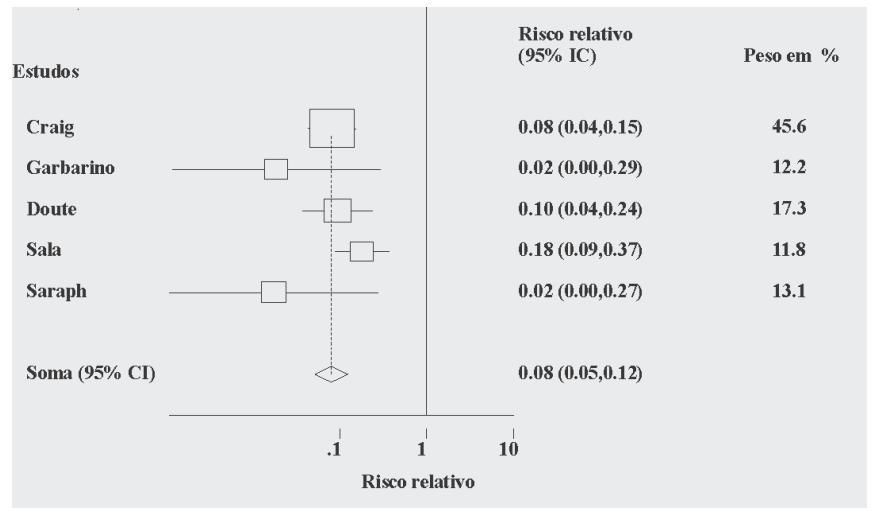

Gráfico 5 - Resultado da análise dos estudos com média de idade maior do que sete anos

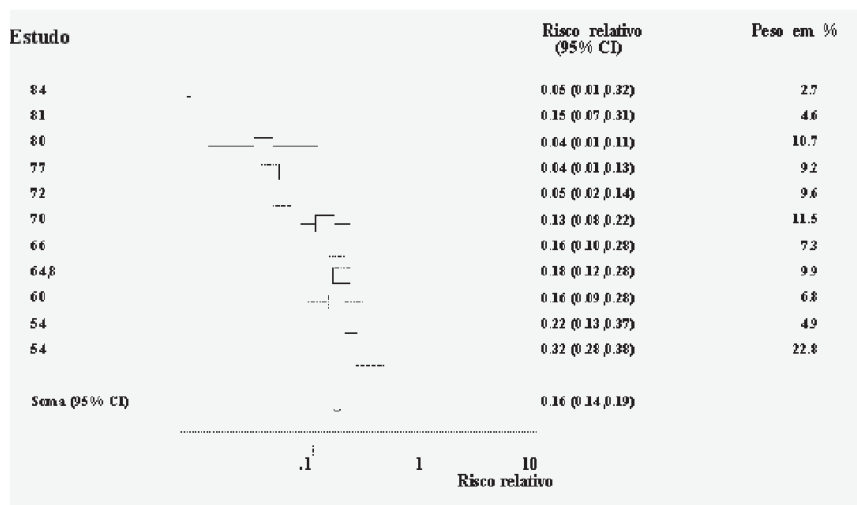

Gráfico 6 - Resultado da análise dos estudos com média de idade menor do que sete anos

A análise gráfica comparativa dos estudos que citam alongamentos realizados no tendão calcâneo mostra soma estatística dos riscos relativos de valor inferior com relação aos estudos que citam alongamentos na junção músculo-tendão, 0,13 e 0,16 , respectivamente (gráficos 7 e 8). A diminuição do risco absoluto é de 0,03 e a diminuição percentual do risco relativo é de $8,13 \%$. A cada 33 pacientes operados prevenimos uma recidiva $(1 / 0,03)$. A longo prazo, este risco tende a aumentar, quando avaliamos o estudo de Olney et al ${ }^{(9)}$ (gráfico 7).

\section{DISCUSSÃO}

Não existem na literatura até 2007 ensaios clínicos randomizados que testem e comparem técnicas para o alongamento do tendão calcâneo. Também não encontramos revisões sistemáticas da literatura que utilizem 


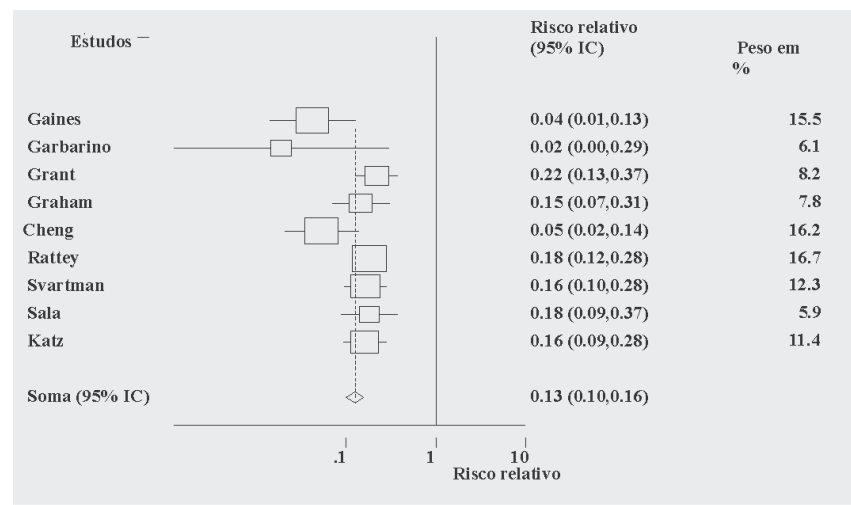

Gráfico 7 - Resultado da análise dos estudos sobre alongamento do tendão calcâneo

a metanálise, como método estatístico de soma dos resultados.

A análise comparativa dos estudos, sem grupo-controle, mostrou menor taxa de recidiva naqueles que realizaram procedimentos cirúrgicos onde é possível o controle da quantidade de alongamento necessário. Garbarino et al não encontraram recidivas em 20 pacientes, nos quais o alongamento do tendão calcâneo com zetaplastia foi assistido por um método geométrico de cálculo da quantidade a ser alongada ${ }^{(10)}$. Saraph et al reproduziram a técnica idealizada por Baumann et al (alongamento controlado da fáscia interna dos músculos gastrocnêmios e solear) em 22 pacientes e também não encontraram recidivas ${ }^{(11-12)}$.

A análise comparativa dos estudos com grupo-controle mostrou menor risco relativo de recidivas, a favor do alongamento do tendão calcâneo, sendo que Lemperg et al encontraram menor recidiva nos pacientes submetidos ao alongamento do tendão calcâneo por zetaplastia $^{(13)}$. Lee et al encontraram o mesmo com a técnica do alongamento fracionado de $\mathrm{Hoke}^{(3,14)}$.

Os estudos com seguimento médio mais longo, isto é, com mais de cinco anos, mostraram que as recidivas começam a aparecer na maioria dos casos após o quinto ano de pós-operatório, com aumento significativo do risco absoluto. Este achado foi observado também por Grant et al e Rattey et al, porém apenas como forma de sugestão e até então sem avaliação estatística. Estes autores também sugeriram em seus estudos que o alongamento no tendão calcâneo por zetaplastia

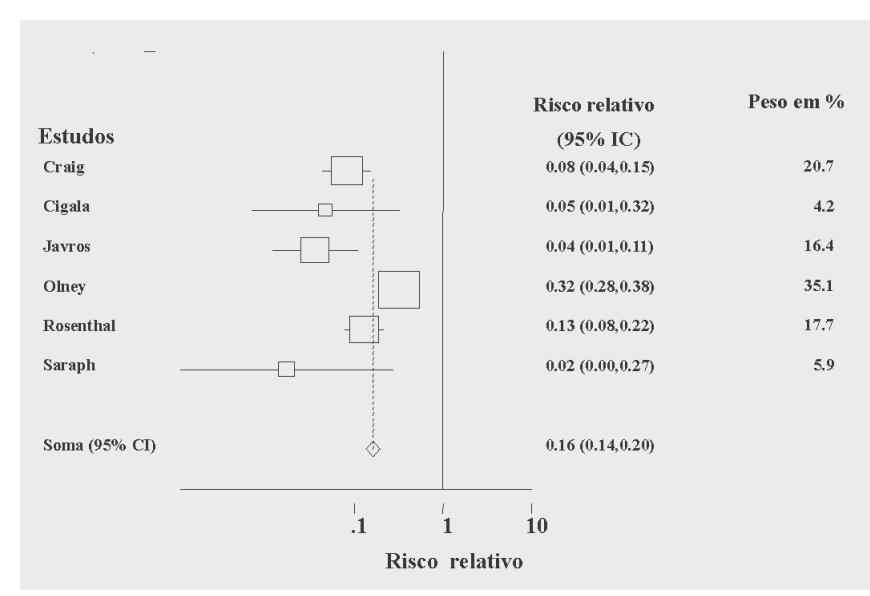

Gráfico 8 - Resultado da análise dos estudos sobre alongamento na junção músculo tendão

tende a ser mais estável, com relação a recidivas, num seguimento em longo prazo $^{(15-16)}$.

A análise dos estudos de procedimentos realizados na junção musculotendínea mostram aumento do risco absoluto de recidivas e a longo prazo este risco tende a aumentar. Olney et al mostraram que num seguimento médio de sete anos e sete meses a redução do risco de recidivas dos pacientes operados pela técnica de Baker et al foi de $68 \%{ }^{(9)}$. Em contrapartida, Grant et al seguiram seus pacientes por 10 anos e mostraram que o alongamento feito no tendão calcâneo gera número menor de recidivas ao longo dos anos ${ }^{(15)}$.

A análise dos estudos de procedimentos realizados no tendão calcâneo mostra soma dos riscos relativos de menor valor numérico com relação aos estudos de procedimentos na junção, porém com diminuição pequena no risco absoluto de recidivas. Estes dados mostram que esta diferença, que em valor percentual representa $8,13 \%$, justifica a preferência pelo alongamento no tendão calcâneo. Devemos aguardar por estudos com maior tempo médio de seguimento para que esta diferença aumente.

Os estudos em que a média de idade dos pacientes, no momento da cirurgia, foi superior aos sete anos mostram menor soma dos riscos relativos, quando comparados ao grupo com média de idade inferior aos sete anos, evidenciando a diminuição significativa do risco absoluto de recidivas ${ }^{(10-11,17-19)}$. Borton et al estabeleceram a idade abaixo dos oito anos em pacientes he- 
miplégicos como fatores de risco para as recidivas da deformidade em eqüino ${ }^{(20)}$.

Em muitas situações estudos randomizados controlados não estão viáveis e somente dados de estudos observacionais estão disponíveis. Apesar das metanálises restritas aos estudos prospectivos randomizados serem a preferência inicial, o número de metanálises publicadas sobre estudos observacionais vem aumentando substancialmente nas últimas quatro décadas ${ }^{(21)}$. Trabalhos sobre ortopedia pediátrica limitam-se, em sua maioria, a modelos retrospectivos. Winter, em comentário intitulado "A falência da análise de resultados computados em curto prazo, em ortopedia pediátrica", comenta sobre a dificuldade de avaliar o resultado de certas cirurgias como a correção de quadris displásicos em adolescentes, devido à freqüente impossibilidade de realizar estudos prospectivos randomizados e controlados, tendo como grupo-controle pacientes não tratados e, portanto, de difícil seguimento por longo prazo. Dessa forma este autor propõe a publicação de estudos em ortopedia pediátrica somente quando os pacientes estiverem adultos ${ }^{(22)}$.

Existe na literatura uma revisão sistemática, porém sem metanálise, que não avalia a qualidade dos estudos e não tem critérios de inclusão e, dessa forma, não leva a conclusões estatisticamente válidas ${ }^{(23)}$.

Dos 20 estudos incluídos em nossa avaliação, 16 não têm grupo-controle ${ }^{(3,9-11,13,15-19,24-33)}$. Utilizou-se, então, a proposta ou solução de Light et al que sugere a criação de grupos-controle fictícios como se fossem pacientes não tratados e, portanto, com $100 \%$ de recidi$\mathrm{va}^{(7)}$. Fez-se o cálculo dos riscos relativos de cada um dos estudos, já que esta prática não é comum em estudos observacionais do tipo análise de resultados. Mais da metade dos estudos incluídos tem boa validade interna e conseqüente boa qualidade. Estudos de maior peso estatístico têm relação diretamente proporcional ao tamanho da amostra de pacientes. Este achado mostra que a decisão de limitar o número de pacientes nos critérios de seleção tem embasamento estatístico e matemático.

O benefício do tratamento cirúrgico do pé eqüino na paralisia cerebral fica evidente quando analisamos a soma estatística dos riscos relativos de cada estudo sem grupo-controle igual a 0,14 , significando que houve redução de $86 \%$ no risco de recidivas nestes 16 estu$\operatorname{dos}^{(9-11,15-19,26-33)}$. Procurou-se selecionar estudos com no mínimo 20 pacientes e com seguimento mínimo de dois anos. Fez-se também a análise da heterogeneidade dos estudos incluindo-os em três subgrupos: seguimento médio maior e menor que cinco anos, técnica cirúrgica e média de idade maior e menor do que sete anos no momento da cirurgia.

A capacidade de generalização dos resultados obtidos é grande, pois a questão inicial foi respondida e os dados obtidos foram submetidos à apreciação estatística. Dessa forma, as recomendações podem ser formuladas de maneira apropriada e com o devido embasamento científico.

Os alongamentos no tendão calcâneo resultam em risco relativo de recidivas igual a 0,13 . Nos alongamentos na junção musculotendínea este valor é de 0,16 . A interpretação do valor do risco relativo de 0,13 é feita da seguinte forma: subtrai-se este valor do numeral um e se obtém a percentagem de $87 \%$ de redução do risco relativo de recidivas. O mesmo é feito para o valor de 0,16 nos alongamentos na junção musculotendínea e chega-se ao valor de $84 \%$. Apesar da redução do risco relativo ser pequena e pouco significativa, o alongamento no tendão calcâneo tende a ser mais seguro quanto ao risco de recidiva. Observando e analisando o gráfico 1, que mostra o resultado da metanálise dos estudos com grupo-controle, nota-se que o alongamento no tendão calcâneo produz uma soma da redução relativa de recidivas de $26 \%$.

Os pacientes operados após os sete anos apresentaram redução relativa nas recidivas de $92 \%$ e de $84 \%$ abaixo dos sete anos. Fica claro, então, que os pacientes devem ser operados preferencialmente após os sete anos de idade. Porém, não devemos menosprezar as deformidades em crianças mais jovens e indicar sempre na ocasião do diagnóstico o tratamento não cirúrgico. Se a deformidade for grave e a criança de baixa idade, podemos indicar o tratamento cirúrgico informando à família do paciente sobre a necessidade de outros procedimentos no futuro.

Os pacientes com tempo de seguimento maior do que cinco anos apresentam um aumento absoluto do 
risco de recidivas de 0,11. Acima dos cinco anos a redução do risco relativo é de $82 \%$ e abaixo dos cinco, de $93 \%$. Estes valores mostram que a longo prazo o risco de recidiva aumenta de forma significativa, reforçando a necessidade do seguimento destes pacientes, no mínimo, até a maturidade esquelética.

Apesar da heterogeneidade estatística, a metanálise de estudos observacionais é possível. O fato de os estudos serem heterogêneos não os invalida. Nós superamos esta situação adversa por meio da análise de subgrupo.

Devido à falta de dados nos estudos incluídos, não obtivemos resultados quanto à deformidade em calcâneo; apenas três estudos fazem menção a esta complicação e de forma vaga. Nos estudos incluídos, não há divisão dos pacientes quanto à distribuição topográfica da paralisia cerebral, logo não tivemos a oportunidade de comparar os efeitos e complicações dos alongamentos do tendão calcâneo entre pacientes hemiplégicos e diplégicos. Recomenda-se que todo esforço deve ser dispensado para a publicação de estudos retrospectivos com grupo-controle e com pacientes que já tenham atingido a maturidade esquelética.

\section{CONCLUSÃO}

A zetaplastia para o alongamento do tendão calcâneo tende a ser mais segura. Devido à falta de dados nos estudos incluídos, não obtivemos resultados quanto à deformidade em calcâneo. A pesquisa demonstrou que, sempre que possível, os pacientes devem ser operados após os sete anos de idade. A longo prazo, o risco de recidiva aumenta de forma significativa reforçando a necessidade do seguimento destes pacientes, no mínimo, até a maturidade esquelética.

\section{REFERÊNCIAS}

1. Basset FH 3rd, Baker LD. Equinus deformity in cerebral palsy. In: Adams JP, editor. Current practice in orthopaedic surgery. St. Louis: Mosby; 1966. p. 59-74.

2. Ziv I, Blackburn N, Rang M, Koreska J. Muscle growth in normal and spastic mice. Dev Med Child Neurol. 1984;26(1): 94-9.

3. Lee CL, Bleck EE. Surgical correction of equinus deformity in cerebral palsy. Dev Med Child Neurol. 1980;22(3):287-92.
4. Horstmann Bleck EE. Orthopaedic management in cerebral palsy. London: MacKeith Press; Philadelphia: J.B. Lippincott; 1987. p. 486. [ Série: Clinics in Developmental Medicine: n.99/ 100].

5. Goldstein M, Harper DC. Management of cerebral palsy: equinus gait. Dev Med Child Neurol. 2001;43(8):563-9.

6. Petitti DB. Meta-analysis, decision analysis, and costeffectiveness analysis: methods for quantitative synthesis in medicine. 2nd ed. New York: Oxford University Press; 2000. p. 306.

7. Light RJ, Pillemer DB. Quantitative procedures. In: Light RJ, Pillemer DB. Summing up: the science of reviewing research. Cambridge, Mass.: Harvard University Press; 1984. p. 50-103.

8. Intercooled Stata [computer program]. Version 8 for windows. Texas: Stata Corp LP; 2003. 1 disk.

9. Olney BW, Williams PF, Menelaus MB. Treatment of spastic equinus by aponeurosis lengthening. J Pediatr Orthop. 1988; $8(4): 422-5$.

10. Garbarino JL, Clancy M. A geometric method of calculating tendo Achillis lengthening. J Pediatr Orthop. 1985;5(5):573-6.

11. Saraph V, Zwick EB, Uitz C, Linhart W, Steinwender G. The Baumann procedure for fixed contracture of the gastrosoleus in cerebral palsy. Evaluation of function of the ankle after multilevel surgery. J Bone Joint Surg Br. 2000;82(4):535-40. Comment in: J Bone Joint Surg Br. 2000;82(7):1084-5.

12. Baumann JU, Koch HG, apud Saraph V, Zwick EB, Uitz C, Linhart W, Steinwender G. The Baumann procedure for fixed contracture of the gastrosoleus in cerebral palsy. Evaluation of function of the ankle after multilevel surgery. J Bone Joint Surg Br. 2000;82(4):535-40. Comment in: J Bone Joint Surg Br. 2000;82(7):1084-5.

13. Lemperg R, Hagberg B, Lundberg A. Achilles tenoplasty for correction of equinus deformity in spastic syndromes of cerebral palsy. Acta Orthop Scand. 1969;40(4):507-19.

14. Hoke M. An operation for correction of extremely relaxed flat feet. J Bone Joint Surg. 1931;13:773-83.

15. Grant AD, Feldman R, Lehman WB. Equinus deformity in cerebral palsy: a retrospective analysis of treatment and function in 39 cases. J Pediatr Orthop. 1985;5(6):678-81.

16. Rattey TE, Leahey L, Hyndman J, Brown DC, Gross M. Recurrence after Achilles tendon lengthening in cerebral palsy. J Pediatr Orthop. 1993;13(2):184-7.

17. Craig JJ, van Vuren J. The importance of gastrocnemius recession in the correction of equinus deformity in cerebral palsy. J Bone Joint Surg Br. 1976;58(1):84-7.

18. Douté DA, Sponseller PD, Tolo VT, Atkins E, Silberstein CE. Soleus neurectomy for dynamic ankle equinus in children with cerebral palsy. Am J Orthop. 1997;26(9):613-6.

19. Sala DA, Grant AD, Kummer FJ. Equinus deformity in cerebral palsy: recurrence after tendon Achillis lenghtening. Dev Med Child Neurol. 1997;39(1):45-8. 
20. Borton DC, Walker K, Pirpiris M, Nattrass GR, Graham HK. Isolated calf lengthening in cerebral palsy. Outcome analysis of risk factors. J Bone Joint Surg Br. 2001;83(3):364-70.

21. Stroup DF, Berlin JA, Morton SC, Olkin I, Williamson GD, Rennie D, et al. Meta-analysis of observational studies in epidemiology: a proposal for reporting. Meta-analysis of Observational Studies in Epidemiology (MOOSE) group. JAMA. 2000;283(15):2008-12.

22. Winter RB. The fallacy of short-term outcomes analysis in pediatric orthopaedics. J Bone Joint Surg Am. 1999;81(10): 1499-500. Comment in: J Bone Joint Surg Am. 2001 Apr;83A(4):619-20. J Bone Joint Surg Am. 2001 Apr;83-A(4):620-1.

23. Koman LA, Smith BP, Barron R. Recurrence of equinus foot deformity in cerebral palsy patients following surgery: a review. J South Orthop Assoc. 2003;12(3):125-33, quiz 134.

24. Sharrard WJ, Bernstein S. Equinus deformity in cerebral palsy. A comparison between elongation of the tendo calcaneus and gastrocnemius recession. J Bone Joint Surg Br. 1972;54(2): 272-6.

25. Pierrot AH, Murphy OB. Albert E. Klinkicht Award, 1972. Heel cord advancement. A new approach to the spastic equinus deformity. Orthop Clin North Am. 1974;5(1):117-26.

26. Cigala F, Marmo C, Misasi M. [Baker's operation in correction of spastic equinus deformity of the foot]. Chir Organi Mov. 1979;65(5):519-23. Italian.
27. Gaines RW, Ford TB. A systematic approach to the amount of Achilles tendon lengthening in cerebral palsy. J Pediatr Orthop. 1984;4(4):448-51.

28. Javros JR, Klaaren HE. The Vulpius procedure for correction of equinus deformity in cerebral palsy. J Pediatr Orthop. 1987; 7(2):191-3

29. Graham HK, Fixsen JA. Lengthening of the calcaneal tendon in spastic hemiplegia by the White slide technique. A long-term review. J Bone Joint Surg Br. 1988;70(3):472-5.

30. Rosenthal RK, Simon SR. The vulpius gastrocnemius- soleus lengthening. In: Sussman MD, editor. The diplegic child. Rosemont, IL: American Academy of Orthopaedic Surgeons; 1992. p. 355-64.

31. Cheng JC, So WS. Percutaneous elongation of the Achilles tendon in children with cerebral palsy. Int Orthop. 1993;17(3): $162-5$.

32. Svartman C, Fucs PMMB, Kertzman PF, Oliveira VM, Prieto EAB. Pé eqüino na paralisia cerebral: análise do tratamento. Rev Bras Ortop. 1994;29(1/2):33-6.

33. Katz K, Arbel N, Apter N, Soudry M. Early mobilization after sliding achilles tendon lengthening in children with spastic cerebral palsy. Foot Ankle Int. 2000;21(12):1011-4. 